\title{
Titanium Nitride as Light Trapping Plasmonic Material in Silicon Solar coll
}

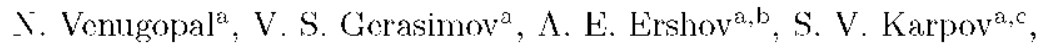 \\ S. P. Polyutovia

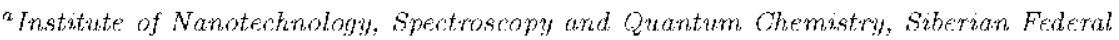 \\ University, Krasnoyarsk, 660041, Russio \\ ${ }^{b}$ Institute of Computational Modeling, Federal Rescarch Center KSC SB RAS Krasnoyarsk, \\ 660036. Russia \\ ¿L.V. Kirensky Institute of Physics, Federat Research Center KSC SB R.S, 660036. \\ Krasnoyarsk, Russiu
}

\begin{abstract}
Light trapping is a crucial prominence to improve the efficiency in thin film solar cells. However, last fow yoars, plasmonic basod thin film solar cells shows potential structure to improve efficiency in photovoltaics. In order to achicve the high efficiency in plasmonic based thin film solar cells, traditionally noble metals like Silver (Ag) and Gold (Au) are extensively used due to their ability to localize the light in manoscale structures. In this paper, we numerically demonstrated the absorption enhancement, due to the incorporation of novel plasmonic TiN nanoparticles on thin film Silicon Solar cells. Absorption enhancement significantly affected by Tix plasmonic nanoparticles on thin film silicon was studied using Finite-Difference-Time-Domain Method (FDTD). The optimal absorption enhancement 1.2 was achieved for TiN nanoparticles with the diameter of $100 \mathrm{~nm}$. 'The results show that the plasmonic effect significantly dominant to achieve maximum absorption enhancement $g(\lambda)$ at longer wavelengths (red and near infrared) and as comparable with Au nanoparticle on thin film silicon. The absorption enhanement can be tuned to the desired position of solar spectrum by adjusting the size of TiN nanoparticles. Fifled, of nanoparticle diameters on the absorption enhancement was also thoroughly
\end{abstract}

\footnotetext{
Corresponding author

Email address: venu369ggmail .com ( V. Venugopal)
} 
analyzed. The numerically simulated results show that TiN can play the similar role as gold nanoparticles on thin film silicon solar cells. Furthermore, TiN plasmonic material is cheap, abundant and more Complementary Metal Oxide Semiconductor (CMOS) compatible material than traditional plasmonic metals like $\mathrm{Ag}$ and $\mathrm{Au}$, which can be easy integration with other optoelectronic devices.

Keywords: plasmonics, titanium nitride, photovoltaics

\section{Introduction}

Nanoplasmonic applications and technologies have grown tremendously over the last few years [1, 2, 3, 4, 5]. One of the most attractive applications of plasmonics is the efficiency enhancement in photovoltaic devices [6, 7]. Recently, various approaches based on light scattering at rough surfaces such as antireflection coating to minimize reflective losses, highly reflective back contacts, texturing of surfaces and interfaces, patterned black silicon surfaces and using layers with matching refractive index to preferentially scatter light into the active layer have all been used to optimize light absorption [8, 9, 10, 11]. However, these techniques involve high processing cost leaving a need for other less expensive light trapping techniques to improve the efficiency at low cost [12].

Light trapping by plasmonic materials involves a strong interaction of light with conduction electrons in metal nanoparticles with an appropriate size and shape integrated to the surface. In this process, incident light stimulates the oscillation of conduction electrons at interfaces containing metal nanoparticles or nanostructures of subwavelength size. When the natural frequency of collective oscillating electrons matches that of incoming light, a localized surface plasmon resonance (LSPR) occurs. At plasmonic resonance, light is preferentially scattered by the nanoparticles decorating the surface of solar cell into solar cell's actively absorbing layer and improves free carrier generation. Stuart and Hall et al. instigated the pioneering work, the effect of plasmonics on silicon photodiodes that fascinated the interest of solar cell researchers. They demonstrated an enhancement in the photocurrent of a factor 18 could be achieved for $160 \mathrm{~nm}$ 
thick silicon on insulator photo-detector at the $800 \mathrm{~nm}$ wavelength from approximately $100 \mathrm{~nm}$ sized silver particles on the surface of device [13]. Following Schaadt et al. obtained enhancement up to $80 \%$ at wavelength around $500 \mathrm{~nm}$ by depositing gold nanoparticles on highly doped wafer-based solar cells [14. Pillai et al. deposited silver particles on $1.25 \mathrm{~mm}$ thick silicon-on-insulator solar cells and planar wafer based cells, and observed a significant enhancement of the absorption for both thin-film and wafer-based structures at wavelengths close to the band gap of Si. They reported a sevenfold enhancement of photocurrent for wafer-based cells at $1200 \mathrm{~nm}$ and up to 16-fold enhancement at $1050 \mathrm{~nm}$ for $1.25 \mathrm{~mm}$ thin silicon-on-insulator (SOI) cells [6, 15]. Beck et al. showed that through the modification in local environment of silver nanoparticles, the optical absorption in the underlying silicon wafer can be enhanced five times and the external quantum efficiency by a factor of 2.3 at a wavelength of $1100 \mathrm{~nm}$ [16]. Later Moulin et al. reported improved light absorption from silver nanoparticles integrated on the rear side of thin film microcrystalline solar cells [17. The effect of LSPR scattering has been reported not only for the case of silicon but also for other semiconductors; (e.g. GaAs) solar cells [18. P. Spinelli et al. observed the absorption enhancement using silver nanoparticles on Crystalline Silicon and Amorphous silicon structures [7. In addition, widely studied by using plasmonics nanoparticles improved the light absorption with Transparent conductive oxides (TCO) of top/bottom thin film solar cells structures has been improved [7, 19, 20, 21].

Traditionally, noble metallic nanoparticles like gold $(\mathrm{Au})$ and silver $(\mathrm{Ag})$ are extensively used for plasmonic based thin film solar cells [20, 22]. These noble metals are very prominent for their plasmonic effects in the wavelength range of the solar spectrum. However these materials were suffering major limitations such as lossy behavior, high cost, low thermal and oxidation stability, and incompatibility with industrial processes [23.

In recent years, transition metal nitrides set the new trend in novel plasmonic materials having great attention and specifically, Titanium Nitride and Zirconium Nitride are known for their unique properties similar in the behavior 
of metals 24]. G. Naik and V. Shalaev et. al. group have been proposed and experimentally demonstrated transition metal nitrides like TiN as an alternative class of materials for practical plasmonic applications in the visible and infrared range [25, 26, 27, 28. This material exhibits optical properties analogous to that of gold for plasmonic applications [29, 30]. Recently, Khalifa et al. theoretically demonstrated the absorption enhancement by incorporating the TiN nanoparticles in Amorphous Silicon Heterojunction solar cells and Organic solar cells [19, 26, 5. Therefore, Titanium Nitride (TiN) has sparked as a potential candidate for broadband absorption covering the entire visible-infrared regions of the electromagnetic spectrum with a surplus benefits such as low loss and cost, high thermal and oxidative stability, unlike Au and Ag. TiN is fully compatible with the existing CMOS fabrication techniques in addition TiN exhibits biocompatibility [19, 31, 32, 33.

In this paper, we numerically studied how the plasmonic material TiN nanoparticles influence the light trapping and absorption enhancement in thin film silicon solar cells. The absorption enhancement of silicon layer in the solar cell with spherical TiN and Au nanoparticles of different sizes has been discussed in detail and compared by using Lumerical FDTD Solutions. A detailed analysis and interpretation have been made for the absorption enhancement. Our results show that the optimal absorption enhancement up to $20 \%$ for TiN Nanoparticle at diameter $100 \mathrm{~nm}$ in the visible region (600-1100 nm) opens up the possibility of its use in thin film silicon solar cells. Our further experimental investigation is in this direction to verify and improve the absorption enhancement with a combination of different $\mathrm{Ag} / \mathrm{Au} / \mathrm{TiN}$ nanoparticle parameters like diameter, period, height and surface coverage on thin film silicon solar cell. Simulation results show that TiN has comparable performance with $\mathrm{Au}$ and in addition this material provides its own indispensable advantages over $\mathrm{Au}$ and $\mathrm{Ag}$. The insights in this paper suggest that TiN can be a potential plasmonic material for thin film photovoltaics, Visible and infrared applications and optoelectronics. 


\section{Structure and Simulation Method}

For FDTD Simulation, we studied Titanium Nitride (TiN) and Gold (Au) nanoparticles on thin film silicon solar cell of thickness $2 \mu \mathrm{m}$ placed at center of the solar cell as shown in Figure 1. The nanoparticle diameters were varied

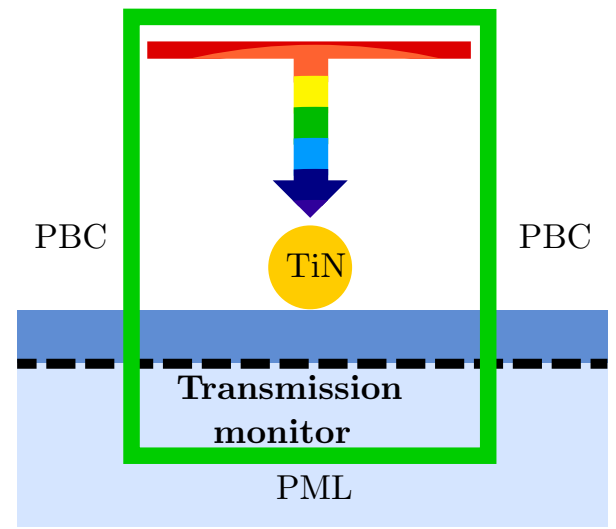

Figure 1: Schematic picture of typical Single Metal nanoparticle placed on thin film Silicon solar ce 11.

from 0 to $200 \mathrm{~nm}$ and the periodic boundary conditions (PBC) in the lateral direction and also perfectly matched layer (PML) were used. A plane wave was used to incident normally on thin film solar cell with the wavelength range from $300 \mathrm{~nm}$ to $1100 \mathrm{~nm}$. Absorbed power in the silicon for individual nanoparticle diameters $\left(P_{a b s}\right)$ was determined according to the FDTD conditions and finding the optimum value of the nanoparticle size by using Sweep parameter. The absorption enhancement with optimum nanoparticle diameter on silicon solar cell with and without nanoparticles was calculated. The dielectric constants of Gold, TiN and Si were taken from Palik [34. The efficiency of solar cell was estimated through our numerical computations results in terms of standard solar cell optical performance parameters like Quantum Efficiency (QE) [25, 35, 36]

$$
Q E(\lambda)=\frac{P_{a b s}(\lambda)}{P_{\text {inc }}(\lambda)}
$$

Where $P_{a b s}(\lambda)$ and $P_{i n c}(\lambda)$ are the power of the absorbed light and incident light within the Si Solar cell respectively. Using the QE, IQE defined as the 
ratio of amount of charges collected by the solar cell to the number of photons per second absorbed and as written as follows [35;

$$
I Q E=\frac{\int \frac{\lambda}{h c} Q E(\lambda) I_{A M 1.5}(\lambda) d \lambda}{\int \frac{\lambda}{h c} I_{A M 1.5}(\lambda) d \lambda}
$$

Where $\mathrm{h}$ is Planck's constant, $\mathrm{c}$ is the speed of light in the free space and $I_{A M 1.5}(\lambda)$ is the AM 1.5 solar spectrum.

Equations (1) and (2) can be modified further to understand the light absorption efficiency of solar cells with metal nanoparticles is enhanced compared to bare solar cells. The absorption enhancement spectrum $g(\lambda)$ for the wavelength range $300 \mathrm{~nm}$ to $1100 \mathrm{~nm}$ as written as follows;

$$
g(\lambda)=\frac{Q E_{N P}(\lambda)}{Q E_{\text {bare }}(\lambda)}=\frac{P_{N P}(\lambda)}{P_{\text {bare }}(\lambda)}
$$

$g$ was calculated for each nanoparticle diameters simulated via the computation of quantum efficiencies.

$$
G=\frac{I Q E_{N P}}{I Q E_{\text {bare }}}=\frac{\int \lambda P_{N P}(\lambda) I_{A M 1.5}(\lambda) d \lambda}{\int \lambda P_{\text {bare }}(\lambda) I_{A M 1.5}(\lambda) d \lambda}
$$

Where $G$ is the absorption enhancement factor, $P_{N P}(\lambda)$ and $P_{\text {bare }}(\lambda)$ represents the light absorption of the silicon layer with and without nanoparticle at the wavelength.

\section{Results and discussion}

\subsection{Results}

The present work demonstrates the effect of incorporating TiN nanoparticles on thin film Silicon solar cell with the varying diameter in optical properties characterized by LSPR. As shown in Figure 2, the absorption peak at wavelength position at $440 \mathrm{~nm}$ for with and without TiN nanoparticles on thin film silicon. Figure 2 shows that from diameter $50 \mathrm{~nm}$ to $200 \mathrm{~nm}$, the absorption increases and then decreases with increment the size of spherical TiN nanoparticles on thin film silicon. Initially, the absorption increases with diameter of 
TiN nanoparticles up to $100 \mathrm{~nm}$ and then decreases. The maximum absorption value is nearly 0.995 for TiN nanoparticle with the diameter of $100 \mathrm{~nm}$. It is due to directly transmitted light at the silicon interface and reradiated or scattered incident light by the TiN nanoparticle are in phase which results enhancement in the absorption [37]. Meanwhile the absorption peak red-shifts and also broadening in wavelength range with the increment of TiN nanoparticle diameter from 40 to $200 \mathrm{~nm}$. The results observe that the absorption ratio of silicon with spherical nanoparticles of different diameters from 40 to $170 \mathrm{~nm}$ is larger than that of bare silicon in the visible light wavelength range. When the diameter is from $110 \mathrm{~nm}$ to $200 \mathrm{~nm}$, the absorption decreases and becomes much lower than that of the bare silicon, it is due to phase shift between scattered and incident field which results in the reduction of absorption. In addition, TiN nanoparticles absorb more light as the diameter increases which results in poor absorption of silicon [36. In Figure 2 it can also observe that in the near infrared wavelength range, the absorption peaks are enhanced which influenced by forward scattering effects due to LSPR [36, 38].

Figure $3 \mathrm{a}$, and $3 \mathrm{p}$, show that the absorption enhancement $g(\lambda)$ spectrums

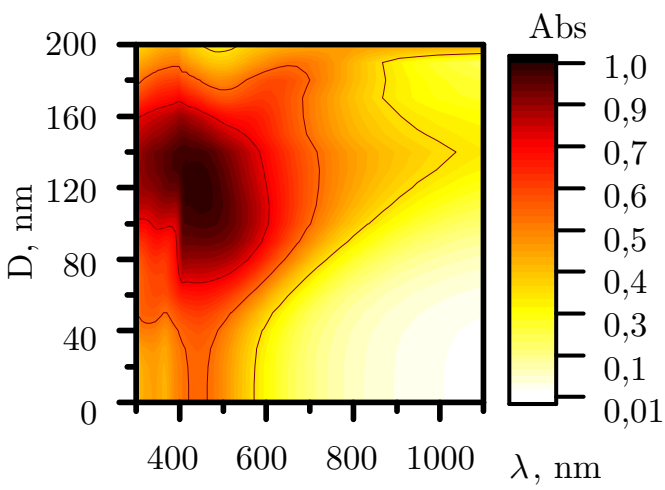

Figure 2: The absorption Spectra for different particle Diameter $-\operatorname{Abs}(\lambda, D)$

with varying the diameters of $\mathrm{TiN}$ and $\mathrm{Au}$ Nanoparticles. The absorption enhancement is not constant along the whole solar spectrum due to LSPR. The graph clearly shows considerable absorption enhancement $g(\lambda)$ about longer 
wavelength from $600 \mathrm{~nm}$ to $1100 \mathrm{~nm}$ region by enhanced forward scattering due to LSPR [35, 14, 39]. Figure 3a shows, Initially the $g(\lambda)$ enhances with TiN nanoparticles size from $40 \mathrm{~nm}$ to $110 \mathrm{~nm}$ about in higher wavelength range $600 \mathrm{~nm}$ to $1100 \mathrm{~nm}$ and $g(\lambda)$ quenches at a shorter wavelength from $300 \mathrm{~nm}$ to $600 \mathrm{~nm}$ and also redshift of absorption with broadening. Reduction of absorption is due to strong parasitic light absorption near the LSPR, where $40 \%$ of the incident energy is dissipated at the shorter wavelength range from 300 to $600 \mathrm{~nm}$ [40, 41] as shown in Figures 3a and 3p. In addition destructive interference between an incident and scattered light are not in phase and also parasitic absorption of higher order resonance modes [38, 42, 43. The maximum enhancement of $g(\lambda)$ at peak value 1.2 at a higher wavelength. Furthermore, when the TiN nanoparticles size increase from $110 \mathrm{~nm}$ to $200 \mathrm{~nm}$, the absorption enhancement $g(\lambda)$ is decreased at the higher wavelength range and also in shorter wavelength range but absorption continuous to redshift with broadening is due to localized surface plasmon resonance.

Figure 3 p shows similar to TiN nanoparticles, initially the $g(\lambda)$ enhances with $\mathrm{Au}$ nanoparticles diameter from $40 \mathrm{~nm}$ to $100 \mathrm{~nm}$ about higher wavelength range $600 \mathrm{~nm}$ to $1100 \mathrm{~nm}$ is due to the forward scattering due to LSPR [35, 14, 39]. Meanwhile $g(\lambda)$ quenches at a shorter wavelength from $300 \mathrm{~nm}$ to $600 \mathrm{~nm}$ and absorption redshift with broadening is due to the reduction in absorption at shorter wavelengths [6, 44, 45]. The maximum enhancement of $g(\lambda)$ at peak value 1.4. Further absorption enhancement $g(\lambda)$ is decreased at higher wavelength range with an increment of TiN nanparticles diameter above $110 \mathrm{~nm}$. The absorption enhancement $g(\lambda)$ also decreases in shorter wavelength range (300 $\mathrm{nm}$ to $600 \mathrm{~nm}$ ) but redshift with broadening due to LSPR. In comparison Figure 5(a), (b) show that the light absorption distribution profile in Silicon with TiN and Au metal nanoparticles and light propagates in silicon film through shortest path [46]. Figure 4 shows the Enhancement Factor (G) varying with metal nanoparticle diameters of both $\mathrm{TiN}$ and $\mathrm{Au}$. It is observed that Enhancement Factor $(\mathrm{G})$ of TiN comparable to Au with an increment of nanoparticle diameters. It clearly shows that absorption enhancement $g(\lambda)$ at 


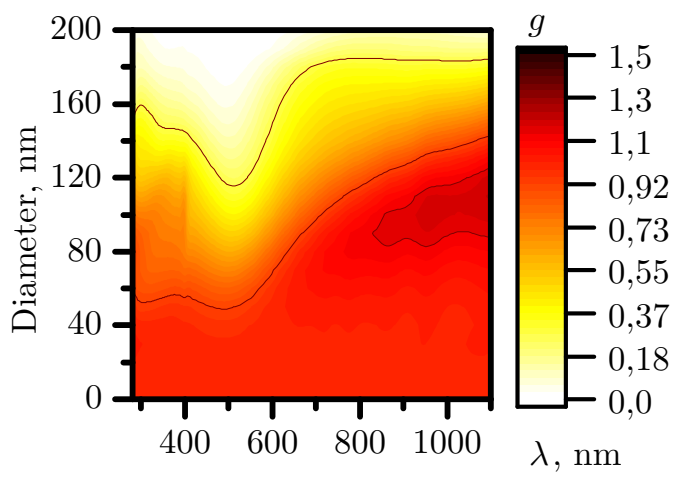

(a)

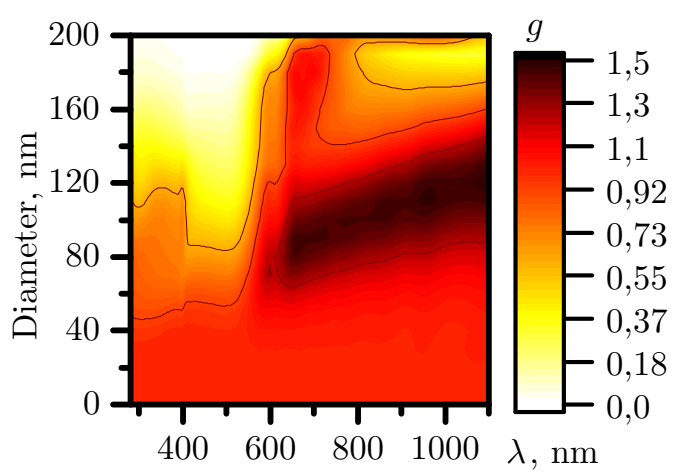

(b)

Figure 3: The absorption enhancement $g(D, \lambda)$ of thin film silicon with different nanoparticle diameters, (a) - TiN, (b) - Au.

a longer wavelength $(600 \mathrm{~nm}$ to $1100 \mathrm{~nm})$ which did not provide a good overall effect to enhance the efficiency of thin film silicon solar cell [19]. Further optimization is required to fully investigate and maximize the efficiency with a combination of different TiN nanoparticle parameters like diameter, period, height and surface coverage on thin film silicon solar cell. The enhancement Factor $(G)$ was reported maximum value with the combination of different TiN nanoparticle diameters, height and periods [25]. 


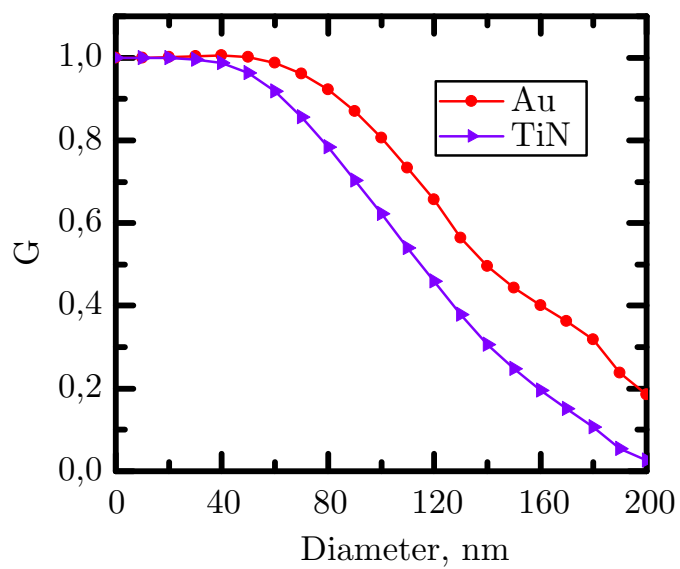

Figure 4: The enhancement Factor $\mathrm{G}$ with different TiN and Au nanoparticle diameters.

\subsection{Discussion}

Numerical results were presented in preceding section the absorption enhancement $g(\lambda)$ spectra clearly demonstrate the TiN nanoparticles and $\mathrm{Au}$ Nanoparticles on silicon. In both spectra Figure $3 \mathrm{a}$, and $3 \mathrm{~b}$, it is observed that a plasmonic $\mathrm{TiN} / \mathrm{Au}$ nanoparticle enhances the absorption enhancement $g(\lambda)$ in the broad range about $600 \mathrm{~nm}$ to $1100 \mathrm{~nm}$ of nanoparticle diameter $100 \mathrm{~nm}$. the Absorption enhancement $g(\lambda)$ increment is ascribed as following reasons;

This is due to the low absorption and strong forward scattering of light by plasmonic ( $\mathrm{TiN} / \mathrm{Au})$ nanoparticles at optimum diameter $100 \mathrm{~nm}$ [4]. The forward scattering can contribute to enhancing absorption, whereas the backward scattering can prohibit to absorption. It is well known that around $100 \mathrm{~nm}$ size of spherical plasmonic nanoparticles usually having the large cross-section area of scattering. The further increment of metal nanoparticle size, higher order mode excitation will take place, which light scatter can enhance or decrease the light trapping efficiency into the substrate depending upon the order of modes excited [22, 44, 45, 13, 48. Generally all modes excited in metal nanoparticle are responsible for forward scattering but alternative modes are 


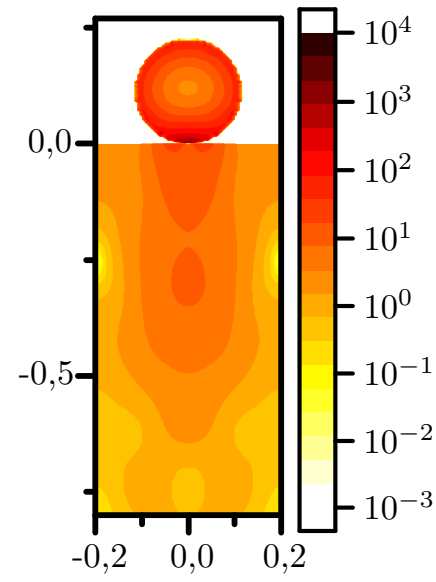

(a)

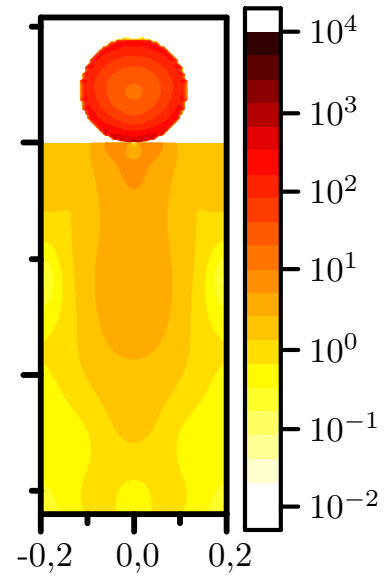

(b)

Figure 5: The relative absorption distribution per unit volume in silicon with $\mathrm{Au}-$ (a) and TiN - (b).

responsible for backscattering like a dipole and octupolar modes are responsible for backscattering [49. The absorption enhancement $g(\lambda)$ with the optimum size of the metal nanoparticle $100 \mathrm{~nm}$ in our case, the forward scattering efficiency of the particle enhances due to the excitation of quadrupole resonances. In our structure, we used spherical nanoparticle because it is well known that higher efficiency of spherical nanoparticles for the forward scattering light over a broader spectrum. Considering the spherical shape of the metal nanoparticle, for a spheroid metal nanoparticle with radius $(\mathrm{R})$ very much smaller than the incident wavelength $(R \leq \lambda)$, applied field would be uniform across the particle. Physically, free electrons inside the particle will all move in coherence in response to the uniform field building upon the polarization of charges on the particle surface enhancing the forward scattering [48, 50, 51]. In addition, Forward scattering also changes depending upon the surrounding medium. When the metal nanoparticle is surrounded by a homogeneous medium, scattering is equal into the forward and backward directions. If the plasmonic nanoparticle 
deposited between the air-glass interface then preferential scattering would be into the glass due to its higher refractive index [52, 53]. This effect is the preferential scattering of light at the particle resonance into the high-index substrate like silicon [7]. K. R. Catchpole and A. Polman et al. investigated for more realistic Ag nanoparticles and found that the fraction of light scattered into the substrate was highly dependent on the three dimensional shape of the nanoparticle [22, 52, 53]. Besides this antireflection effect, constructive interference between the directly transmitted light at the Silicon interface and reradiated or scattered incident light by the nanoparticles when they are in phase which also results the absorption enhancement [16, 20, 54, 55].

When the nanoparticle diameter increases beyond $100 \mathrm{~nm}$ the absorption enhancement $g(\lambda)$ gradually decreases. Absorption enhancement $g(\lambda)$ decrement is ascribed as following reasons;

The absorption broadening with redshift due to the size of nanoparticle increases results in the inhomogeneous polarization of the plasmonic nanoparticles by the electromagnetic field and thus the higher order modes get excited (quadrupole, octopole and hexopole). The presence of these higher oscillation modes shifts the absorption shifts to longer wavelength [56]. The phase coherence of absorption gets destroyed due to the interaction between dipole and higher order oscillatory motions of the electrons. This may account for observed reduced absorption enhancement, the redshift of absorption with increased nanoparticles size. In addition, destructive interference between incident and scattered light are not in phase which results the reduction in absorption enhancement.

The maximum absorption enhancement $g(\lambda)$ of $20 \%$ is achieved when the TiN nanoparticles diameter is about $110 \mathrm{~nm}$ and $40 \%$ of enhancement is achieved in case $\mathrm{Au}$ Nanoparticles about $100 \mathrm{~nm}$. The plasmonic effect seems to be dominant as the maximum of absorption enhancement $g(\lambda)$ occurs at a longer wavelength (red and near infrared). As particle diameter increase, initially the absorption enhancement $g(\lambda)$ grows at a longer wavelength and then decreases and in a meanwhile reduction in absorption at shorter wavelengths due to LSPR. 
This is an agreement with a plasmonic resonance occurring in the near infrared wavelengths [38, 44, 45]. Absorption enhancement $g(\lambda)$ of TiN is very similar with gold nanoparticles. Comparing TiN and Au performances when the nanoparticles placed on Silicon, it can be deduced that there is no substantial difference in their effects. It was proven that TiN has the potential to replace $\mathrm{Au}$ in certain plasmonic applications. Simulation results show that TiN has comparable performance to $\mathrm{Au}$ in addition to provide indispensable advantages such as its chemical stability and lower cost.

\section{Conclusion}

In conclusion, we proposed and numerically investigated TiN as an alternative plasmonic material for thin silicon solar cells. The influence of TiN nanoparticle diameter on the light absorption enhancement of the thin cell has been studied. The maximum absorption enhancement $g(\lambda)$ of $20 \%$ is achieved when the TiN nanoparticle diameter is about $100 \mathrm{~nm}$ and $40 \%$ of enhancement is achieved in case Au Nanoparticles about $100 \mathrm{~nm}$. The absorption enhancement peak can be tuned to the desired position of solar spectrum by adjusting the size of TiN nanoparticles. Optimized plasmonic TiN nanoparticles on silicon show a comparable and very close enhancement effect to that of Au Nanoparticles. Further investigations will require more understanding and maximize the efficiency with a combination of different TiN nanoparticle parameters like diameter, period, surrounding medium, height and surface coverage on thin film

silicon solar cell. In general, our calculated results shown in this paper suggests that the TiN can also be suitable material in thin film solar cells.

\section{References}

[1] A. V. Zayats, Perspective: A glint of the future, Nature 495 (7440) (2013) S7-S7.

URL http://dx.doi.org/10.1038/495S7ahttp://www.nature.com/ nature/journal/v495/n7440_supp/pdf/495S7a.pdf 
[2] Z. Liang, J. Sun, Y. Jiang, L. Jiang, X. Chen, Plasmonic enhanced optoelectronic devices, Plasmonics 9 (4) (2014) 859-866. doi:10.1007/ s11468-014-9682-7.

[3] A. Polman, Applied physics. plasmonics applied, Science 322 (5903) (2008) 868-9. doi:10.1126/science.1163959.

URL http://www.ncbi.nlm.nih.gov/pubmed/18988831

[4] M. I. Stockman, Nanoplasmonics: past, present, and glimpse into future, Opt. Express 19 (22) (2011) 22029-22106. doi:10.1364/OE.19.022029 URL http://www.opticsexpress.org/abstract.cfm?URI= oe-19-22-22029

[5] S. Magdi, D. Ji, Q. Gan, M. A. Swillam, Broadband absorption enhancement in organic solar cells using refractory plasmonic ceramics, Journal of Nanophotonics 11 (1) (2017) 016001. doi:10.1117/1.jnp.11.016001 URL https://doi.org/10.1117\%2F1.jnp.11.016001

[6] S. Pillai, K. R. Catchpole, T. Trupke, M. A. Green, Surface plasmon enhanced silicon solar cells, Journal of Applied Physics 101 (9) (2007) 093105. doi:10.1063/1.2734885,

[7] P. Spinelli, V. E. Ferry, J. van de Groep, M. van Lare, M. A. Verschuuren, R. E. I. Schropp, H. A. Atwater, A. Polman, Plasmonic light trapping in thin-film si solar cells, Journal of Optics 14 (2) (2012) 024002. doi: $10.1088 / 2040-8978 / 14 / 2 / 024002$.

[8] A. Polman, M. Knight, E. C. Garnett, B. Ehrler, W. C. Sinke, Photovoltaic materials: Present efficiencies and future challenges, Science 352 (6283) (2016) aad4424. doi:10.1126/science.aad4424.

URL http://www.ncbi.nlm.nih.gov/pubmed/27081076

[9] Z. H. Yue, H. L. Shen, Y. Jiang, Antireflective nanostructures fabricated by reactive ion etching method on pyramid-structured silicon surface, Applied Surface Science 271 (2013) 402-406. doi :DOI10.1016/j . apsusc. 2013.01. 
216.

URL <GotoISI> : //WOS:000316787400058

[10] Y. Wang, Y. P. Liu, H. L. Liang, Z. X. Mei, X. L. Du, Broadband antireflection on the silicon surface realized by ag nanoparticle-patterned black silicon, Phys Chem Chem Phys 15 (7) (2013) 2345-50. doi:10.1039/ c2cp44406b.

URL http://www.ncbi.nlm.nih.gov/pubmed/23296192

[11] L. Yang, X. Li, X. Tuo, T. T. Van Nguyen, X. Luo, M. Hong, Alloy nanoparticle plasmon resonance for enhancing broadband antireflection of lasertextured silicon surfaces, Opt Express 19 Suppl 4 (S4) (2011) A657-63. doi:10.1364/0E.19.00A657.

URL http://www.ncbi.nlm.nih.gov/pubmed/21747532

[12] H. Nasser, Z. M. Saleh, E. Ozkol, M. Gunoven, A. Bek, R. Turan, Fabrication of ag nanoparticles embedded in al:zno as potential light-trapping plasmonic interface for thin film solar cells, Plasmonics 8 (3) (2013) 14851492. doi:DOI10.1007/s11468-013-9562-6. URL <GotoISI> ://WOS : 000323276500022

[13] H. R. Stuart, D. G. Hall, Island size effects in nanoparticle-enhanced photodetectors, Applied Physics Letters 73 (26) (1998) 3815. doi:10.1063/ 1.122903 .

[14] D. M. Schaadt, B. Feng, E. T. Yu, Enhanced semiconductor optical absorption via surface plasmon excitation in metal nanoparticles, Applied Physics Letters 86 (6) (2005) 063106. doi:10.1063/1.1855423.

[15] S. Pillai, M. Green, Plasmonics for photovoltaic applications, Solar Energy Materials and Solar Cells 94 (9) (2010) 1481-1486. doi:10.1016/j. solmat.2010.02.046.

URL https://doi.org/10.1016\%2Fj . solmat.2010.02.046 
[16] F. J. Beck, A. Polman, K. R. Catchpole, Tunable light trapping for solar cells using localized surface plasmons, Journal of Applied Physics 105 (11) (2009) 114310. doi:10.1063/1.3140609.

URL https://doi .org/10.1063\%2F1.3140609

[17] E. Moulin, J. Sukmanowski, P. Luo, R. Carius, F. Royer, H. Stiebig, Improved light absorption in thin-film silicon solar cells by integration of silver nanoparticles, Journal of Non-Crystalline Solids 354 (19-25) (2008) 24882491. doi:10.1016/j.jnoncrysol.2007.09.031.

URL https://doi.org/10.1016\%2Fj.jnoncrysol.2007.09.031

[18] K. Nakayama, K. Tanabe, H. A. Atwater, Plasmonic nanoparticle enhanced light absorption in GaAs solar cells, Applied Physics Letters 93 (12) (2008) 121904. doi:10.1063/1.2988288.

URL https://doi.org/10.1063\%2F1.2988288

[19] A. E. Khalifa, M. A. Swillam, Plasmonic silicon solar cells using titanium nitride: a comparative study, Journal of Nanophotonics 8 (1) (2014) 084098. doi:10.1117/1.jnp.8.084098,

[20] S. H. Lim, W. Mar, P. Matheu, D. Derkacs, E. T. Yu, Photocurrent spectroscopy of optical absorption enhancement in silicon photodiodes via scattering from surface plasmon polaritons in gold nanoparticles, Journal of Applied Physics 101 (10) (2007) 104309. doi:10.1063/1.2733649.

[21] H. A. Atwater, A. Polman, Plasmonics for improved photovoltaic devices Nature Materials 9 (3) (2010) 205-213. doi:10.1038/nmat2629

URL http://dx.doi.org/10.1038/nmat2629

[22] M. Schmid, R. Klenk, M. Lux-Steiner, M. Topic, J. Krc, Modeling plasmonic scattering combined with thin-film optics, Nanotechnology 22 (2) (2011) 025204. doi:10.1088/0957-4484/22/2/025204.

URL http://www.ncbi.nlm.nih.gov/pubmed/21135483 
[23] P. Patsalas, N. Kalfagiannis, S. Kassavetis, Optical properties and plasmonic performance of titanium nitride, Materials 8 (6) (2015) 3128-3154. doi:10.3390/ma8063128.

[24] J. B. Khurgin, Replacing noble metals with alternative materials in plasmonics and metamaterials: how good an idea?, Philosophical Transactions of the Royal Society A: Mathematical, Physical and Engineering Sciences 375 (2090) (2017) 20160068. doi:10.1098/rsta.2016.0068

URL https://doi.org/10.1098\%2Frsta.2016.0068

[25] N. Saiprasad, A. Boretti, L. Rosa, S. Castelletto, Novel plasmonic materials to improve thin film solar cells efficiency, in: B. J. Eggleton, S. Palomba (Eds.), Micro-Nano Materials, Devices, and Systems, SPIE-Intl Soc Optical Eng, 2015, pp. 966847-1—966847-10. doi:10.1117/12.2202475. URL http://dx.doi.org/10.1117/12.2202475

[26] A. E. Khalifa, M. A. Swillam, Cheap and efficient plasmonic solar cell, in: A. Freundlich, J.-F. Guillemoles (Eds.), Physics, Simulation, and Photonic Engineering of Photovoltaic Devices III, SPIE-Intl Soc Optical Eng, 2014, pp. 89811R-1-89811R-6. doi:10.1117/12.2040776.

URL http://dx.doi .org/10.1117/12.2040776

[27] S. Murai, K. Fujita, Y. Daido, R. Yasuhara, R. Kamakura, K. Tanaka, Plasmonic arrays of titanium nitride nanoparticles fabricated from epitaxial thin films, Opt Express 24 (2) (2016) 1143-53. doi:10.1364/0E.24. 001143

URL http://www.ncbi.nlm.nih.gov/pubmed/26832498

[28] U. Guler, A. V. Kildishev, A. Boltasseva, V. M. Shalaev, Plasmonics on the slope of enlightenment: the role of transition metal nitrides, Faraday Discussions 178 (0) (2015) 71-86. doi:10.1039/C4FD00208C.

URL http://dx.doi.org/10.1039/C4FD00208Chttp://pubs.rsc.org/ en/content/articlepdf/2015/fd/c4fd00208c 
[29] G. V. Naik, J. L. Schroeder, X. J. Ni, A. V. Kildishev, T. D. Sands, A. Boltasseva, Titanium nitride as a plasmonic material for visible and near-infrared wavelengths, Optical Materials Express 2 (4) (2012) 478-489. URL <GotoISI> ://WOS : 000302957100015

[30] G. V. Naik, V. M. Shalaev, A. Boltasseva, Alternative plasmonic materials: beyond gold and silver, Adv Mater 25 (24) (2013) 3264-94. doi:10.1002/ adma.201205076.

URL http://www.ncbi.nlm.nih.gov/pubmed/23674224

[31] A. A. Herzing, U. Guler, X. Zhou, A. Boltasseva, V. Shalaev, T. B. Norris, Electron energy loss spectroscopy of plasmon resonances in titanium nitride thin films, Applied Physics Letters 108 (17) (2016) 171107. doi:10.1063/ 1.4947442 .

[32] C.-T. Li, S.-R. Li, L.-Y. Chang, C.-P. Lee, P.-Y. Chen, S.-S. Sun, J.-J. Lin, R. Vittal, K.-C. Ho, Efficient titanium nitride/titanium oxide composite photoanodes for dye-sensitized solar cells and water splitting, J. Mater. Chem. A 3 (8) (2015) 4695-4705. doi:10.1039/c4ta05606j.

[33] C. M. Zgrabik, E. L. Hu, Optimization of sputtered titanium nitride as a tunable metal for plasmonic applications, Optical Materials Express 5 (12) (2015) 2786. doi:10.1364/ome.5.002786

URL https://doi.org/10.1364\%2Fome.5.002786

[34] E. D. Palik, List of contributors for volume i, in: E. D. Palik (Ed.), Handbook of Optical Constants of Solids, Academic Press, Burlington, 1997, pp. xv - xvi. doi:http://dx.doi.org/10.1016/B978-012544415-6. $50000-5$

URL http://www.sciencedirect.com/science/article/pii/ B9780125444156500005

[35] D. Zhang, X. Yang, X. Hong, Y. Liu, J. Feng, Aluminum nanoparticles enhanced light absorption in silicon solar cell by surface plasmon resonance, 
Optical and Quantum Electronics 47 (6) (2014) 1421-1427. doi:10.1007/ s11082-014-0103-0.

[36] Z. Yuan, X. Li, H. Jing, Absorption enhancement of thin-film solar cell with rectangular ag nanoparticles, Journal of Applied Sciences 14 (8) (2014) 823827. doi:10.3923/jas.2014.823.827

URL http://dx.doi.org/10.3923/jas.2014.823.827

[37] N. Venugopal, G. Kaur, A. Mitra, Plasmonics effect of ag nanoislands covered n-al:zno/p-si heterostructure, Applied Surface Science 320 (2014) 3042. doi:10.1016/j.apsusc. 2014.09.059.

[38] T. L. Temple, G. D. K. Mahanama, H. S. Reehal, D. M. Bagnall, Influence of localized surface plasmon excitation in silver nanoparticles on the performance of silicon solar cells, Solar Energy Materials and Solar Cells 93 (11) (2009) 1978-1985. doi:10.1016/j.solmat.2009.07.014.

[39] C. L. Tan, S. J. Jang, Y. T. Lee, Localized surface plasmon resonance with broadband ultralow reflectivity from metal nanoparticles on glass and silicon subwavelength structures, Opt. Express 20 (16) (2012) 17448-17455. doi:10.1364/OE.20.017448

URL http://www.opticsexpress.org/abstract.cfm?URI= oe-20-16-17448

[40] Y. Zhang, Z. Ouyang, N. Stokes, B. Jia, Z. Shi, M. Gu, Low cost and high performance al nanoparticles for broadband light trapping in si wafer solar cells, Applied Physics Letters 100 (15) (2012) 151101. doi:10.1063/1. 3703121

[41] Z. Starowicz, G. Kulesza-Matlak, M. Lipiński, Optimization studies on enhanced absorption in thin silicon solar cell by plasmonic silver nanoparticles for the front side configuration, Plasmonics 10 (6) (2015) 1639-1647. doi:10.1007/s11468-015-9996-0. 
[42] B. zeng Li, X. xiao Su, J. quan Lin, X. Gao, J. guo Lei, H. yan Tao, Optical absorption enhancement in solar cells with cylindrical ag nanoparticles by localized surface plasmons, Journal of Modern Optics 58 (9) (2011) 796801. doi:10.1080/09500340.2011.573588,

URL http://dx.doi.org/10.1080/09500340.2011.573588

[43] Y. Zhang, X. Chen, Z. Ouyang, H. Lu, B. Jia, Z. Shi, M. Gu, Improved multicrystalline si solar cells by light trapping from al nanoparticle enhanced antireflection coating, Optical Materials Express 3 (4) (2013) 489-495. doi:10.1364/0ME.3.000489.

URL http://www.osapublishing.org/ome/abstract.cfm?URI= ome-3-4-489

[44] N. Venugopal, A. Mitra, Influence of temperature dependent morphology on localized surface plasmon resonance in ultra-thin silver island films, Applied Surface Sciencedoi:10.1016/j.apsusc.2013.08.062.

[45] N. Venugopal, A. Mitra, Optical transparency of zno thin film using localized surface plasmons of ag nanoislands, Optical Materials 35 (7) (2013) 1467-1476. doi:10.1016/j.optmat.2013.03.003

[46] S. Zhou, X. Hunang, Q. Li, Y. M. Xie, Optimizing two-level hierarchical particles for thin-film solar cells, Optics Express 21 (S2) (2013) A285. doi: 10.1364/oe.21.00a285.

URL https://doi.org/10.1364\%2Foe.21.00a285

[47] Y. Kuo, W.-Y. Chang, H.-S. Chen, Y.-R. Wu, C. C. Yang, Y.-W. Kiang, Surface-plasmon-coupled emission enhancement of a quantum well with a metal nanoparticle embedded in a light-emitting diode, Journal of the Optical Society of America B 30 (10) (2013) 2599. doi:10.1364/josab. 30.002599 .

[48] U. Kreibig, M. Vollmer, Optical Properties of Metal Clusters, Springer Berlin Heidelberg, 1995. doi:10.1007/978-3-662-09109-8

URL http://dx .doi . org/10.1007/978-3-662-09109-8 
[49] I. Diukman, M. Orenstein, How front side plasmonic nanostructures enhance solar cell efficiency, Solar Energy Materials and Solar Cells 95 (9) (2011) 2628-2631. doi:10.1016/j.solmat.2011.05.019.

[50] C. Hägglund, M. Zäch, G. Petersson, B. Kasemo, Electromagnetic coupling of light into a silicon solar cell by nanodisk plasmons, Applied Physics Letters 92 (5) (2008) 053110. doi:10.1063/1.2840676.

[51] C. Hägglund, M. Zäch, B. Kasemo, Enhanced charge carrier generation in dye sensitized solar cells by nanoparticle plasmons, Applied Physics Letters 92 (1) (2008) 013113. doi:10.1063/1.2830817.

[52] K. R. Catchpole, A. Polman, Design principles for particle plasmon enhanced solar cells, Applied Physics Letters 93 (19) (2008) 191113. doi: $10.1063 / 1.3021072$

[53] K. R. Catchpole, A. Polman, Plasmonic solar cells, Optics Express 16 (26) (2008) 21793-21800.

URL <GotoISI> ://WOS:000262200700067

[54] R. Xu, X. Wang, W. Liu, L. Song, X. Xu, A. Ji, F. Yang, J. Li, Optimization of the dielectric layer thickness for surface-plasmon-induced light absorption for silicon solar cells, Japanese Journal of Applied Physics 51 (2012) 042301. doi:10.1143/jjap.51.042301.

URL https://doi.org/10.1143\%2Fjjap.51.042301

[55] Y. Shi, X. Wang, W. Liu, T. Yang, R. Xu, F. Yang, Multilayer silver nanoparticles for light trapping in thin film solar cells, Journal of Applied Physics 113 (17) (2013) 176101. doi:10.1063/1.4803676.

URL https://doi.org/10.1063\%2F1.4803676

[56] A. Patra, A. Manivannan, S. Kasiviswanathan, Spectral response of nanocrystalline zno films embedded with au nanoparticles, Journal of the Optical Society of America B-Optical Physics 29 (12) (2012) 3317-3324. URL <GotoISI> : //WOS:000311990300015 